\title{
IMPROVEMENTS TO THE CATHODE-FOLLOWER RF AMPLIFIER SYSTEM FOR THE LANSCE PROTON STORAGE RING BUNCHER*
}

\author{
J. Lyles, J. Davis, Los Alamos National Laboratory, \\ Los Alamos, New Mexico 87545 USA
}

\begin{abstract}
Los Alamos Neutron Science Center (LANSCE) is upgrading the Proton Storage Ring (PSR) for the Short Pulse Spallation Source, to deliver 200 microamperes $(\mu \mathrm{A})$ of beam current to a new target assembly [1]. Previous operation had been limited to $70 \mu \mathrm{A}$, partly as a consequence of the peak radiofrequency (RF) voltage available from the $h=1$ buncher. After an extensive series of performance tests in 1996, an upgrade was proposed to the 2.8 Megahertz $(\mathrm{MHz})$ cathode-follower RF amplifier system and the ferrite-loaded gap assembly. A new intermediate power amplifier (IPA) was designed and installed at the ring, to eliminate a troublesome oscillation which occurred when the final power amplifier (FPA) was operated in class A. This also increased the drive to the FPA to raise the gap voltage. The cathode-follower FPA was modified to accept the direct connection of a pair of short transmission line feeders from the IPA. The beam gap was replaced with one having larger ceramic insulating sleeves in a simplified mechanical configuration. A new RF coupling loop/ferrite bias conductor was designed to tightly couple the two halves of the ferrite-loaded resonant circuit connected to the gap, to improve waveform symmetry and to reduce the effects of gain differences in the amplifiers. Improvements were made to the amplitude feedback controller and a phase controller was designed and installed. Changes to the utility systems, the controls, and the power supplies completed this upgrade in 1998. A description of the circuit modeling and the hardware changes in the equipment will be presented. As demonstrated, the upgraded RF buncher has delivered the specified performance at LANSCE [2].
\end{abstract}

\section{ORIGINAL RF BUNCHER}

The PSR utilizes a RF barrier system to control longitudinal beam distribution. The 90.2 meter circumference ring has a rotational frequency of 2.79513 $\mathrm{MHz}$, hereafter referred to as $2.8 \mathrm{MHz}$. With a bunch length of 250 nanoseconds (ns), there is a $107 \mathrm{~ns}$ longitudinal space kept clear to allow for beam extraction. The original $\mathrm{h}=1 \mathrm{RF}$ system has been providing up to 13 kilovolts $(\mathrm{kV})$ of $2.8 \mathrm{MHz}$ voltage at the buncher gap.

\subsection{Motivation to Upgrade Buncher}

A series of performance measurements were made to determine shortcomings of the original RF buncher, which could be problematic when the average beam current is increased to $200 \mu \mathrm{A}$. The RF voltage in the present gap would need to be as high as $18 \mathrm{kV}$ at 2.8 $\mathrm{MHz}$. Another goal was to configure the system to allow a second harmonic at $5.6 \mathrm{MHz}$, to improve the bunching factor without reaching a space charge limit in PSR. A plan was developed to upgrade the original buncher and build a second system with the ability to operate at the fundamental or at $\mathrm{h}=2[3]$.

\subsection{Buncher Gap}

The buncher gap is a $1 \mathrm{~cm}$ break in the PSR beam pipe, which is insulated with an outer alumina cylinder. The RF structure consists of a pair of opposing horizontal stubs approximately $79 \mathrm{~cm}$ long. These beam pipes are grounded at the far ends to the enclosure shell, and opencircuited at the center, where the insulator bridges the structure. Twenty water-cooled Philips type $4 \mathrm{H}$ ferrite toroids are stacked on each pipe to raise the inductance, and foreshorten the resonator. Direct current is routed through a bias winding to tune resonance to $2.8 \mathrm{MHz}$. This circuit has $16 \mu \mathrm{H}$ of inductance, and $200 \mathrm{pf}$ of capacitance at the gap, where $150 \mathrm{pf}$ is provided with three fixed vacuum capacitors. At resonance it provides a $5000 \mathrm{Ohm}$ load to the FPA. The fixed capacitors are usable to $80 \mathrm{MHz}$, and pass the higher frequency Fourier components of the image current.

\subsection{Final Power Amplifier}

The PSR may store peak beam currents as high as 35 Amperes ( $\geq 200 \mathrm{~ns}$ bunch, $6.25 \times 10^{13}$ protons), so it is imperative to maintain a very low longitudinal impedance, $|\mathrm{Z}|<20$ Ohms. Shunting the gap with a low resistance was impractical due to the power required. Active feedback around the final amplifier is a standard approach for many circular machines. The PSR buncher uses a cathode-follower (common-anode) FPA. For a cathode-follower using a triode with a high amplification factor, the low frequency output impedance can be estimated as:

$R_{o} \approx \frac{1}{g_{m}}$.This low output impedance loads the resonant gap, and provides a path for the image current through the power triodes. A pair of industrial triodes (EEV part number BW1643J2, formerly Amperex 8918) rated for $240 \mathrm{~kW}$ plate dissipation are used in a push pull arrangement, with the cathodes feeding both sides of the gap with short wide straps. They are mounted under the beamline. The transconductance $\left(g_{m}\right)$ at our operating

${ }^{*}$ Work supported by the US Department of Energy 
point is 0.23 mhos for these power tubes. The low frequency output resistance is calculated to be less than 10 Ohms, across the two cathodes. It is higher at the gap, due to the strap lengths and skin effect. This arrangement has worked successfully since 1984, and is used in the upgraded system as well [4].

It is known that a cathode-follower operating with a tuned grid circuit in the high frequency range can be unsatisfactory, due to possible oscillation in the Colpitts arrangement when the cathode load is capacitive [5,6]. At PSR this is overcome with a $25 \mathrm{Ohm}$ water-cooled resistance in series with the grid of each triode, making a net positive input resistance. Cathode followers have less than unity voltage gain:

$A=\frac{g_{m} R_{k}}{1+g_{m} R_{k}}$, where $R_{k}$ is $1 / 2$ of the resonant load for the push-pull situation, or $2500 \mathrm{Ohms}$ for each triode. The calculated gain is 0.99 , but in hardware it is about 0.93 , because of the series-connected stability resistor at each grid, and circuit drops and losses.

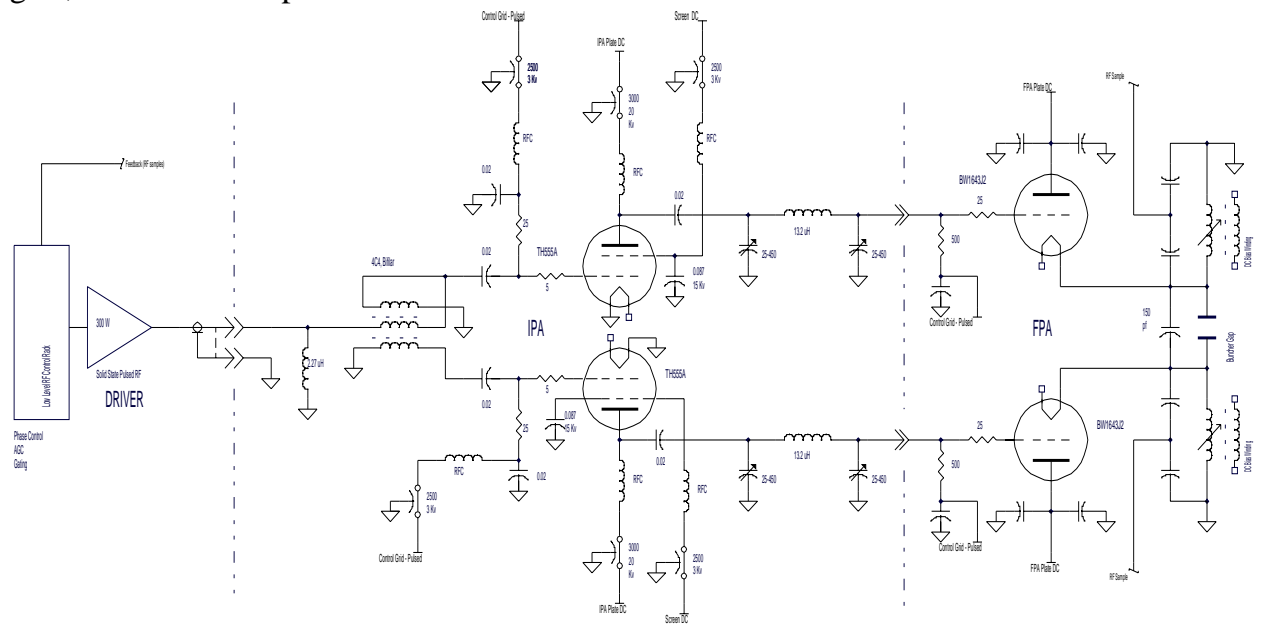

between halves, so the followers acted as independent amplifiers for each half of the total gap voltage.

\section{UPGRADED RF BUNCHER}

\subsection{New Intermediate Power Amplifier}

The replacement IPA is sized to provide all of the voltage gain with $19.5 \mathrm{kV}$ peak output voltage to drive the FPA to provide $18 \mathrm{kV}$ at the gap. Tetrodes are optimal for voltage gain and stage isolation. The parasitic oscillation was eliminated closely coupling a new IPA to the FPA, with the series grid reisistors. This is schematically shown in figure 1. A pair of TH555A tetrodes from Thomson Electron Tubes, rated for $250 \mathrm{~kW}$ plate dissipation, are used in the IPA, operating 180 degrees out of phase. Because we intended to operate them in class $A$, the manufacturer recommended a ring of lossy ferrites around the circumference of the screen to plate ceramic, to suppress higher order UHF modes. We saw no evidence of UHF energy with this socket. Figure $2 \mathrm{a}$ shows the interior of one half of the IPA. The left side of the enclosure houses a duplicate amplifier.

For the IPA stage, we used a 500 Ohm plate loadline so that 20 Amperes of RF current will give us $10 \mathrm{kV}$ peak. Each tube swings $20 \mathrm{kV}$ peak to peak. A 500 Ohm water-cooled shunt resistor loads the grid of each cathode-follower. A pi-network $(\mathrm{Q}=8)$ absorbs the capacitive reactance of each TH555A plate and the input capacitance of each cathode-follower. This low

Figure 1. Simplified Schematic of Improved Buncher

\subsection{Intermediate Power Amplifier}

The original system used two cascaded amplifiers for two stages of voltage amplification. One amplifier used two 4CW25,000A tetrodes, which drove another amplifier with four of the same tubes, near the limit of their voltage rating. They were located in the equipment building above the PSR, interconnected with a pair of Heliax ${ }^{\circledR}$ coaxial cables. These were both fed and terminated in high voltage RF transformers, which then drove the FPA grids. The long cables between stages had reflections responsible for a line resonance at $7 \mathrm{MHz}$, which caused an oscillation in the cathode followers when biased into class A at high quiescent current. Hence, they were operated in class $\mathrm{AB}$, occasionally reaching cutoff and saturation during peak excursions. This hindered the ability to raise buncher voltage and maintain low output impedance. The ferrite cavity lacked RF coupling pass filter provides two tunable elements for compensation of any phase shift in each amplifier. An electric clutch arrangement was designed to mechanically lock the capacitors on each half of the IPA together, to hold the 180 degree phase offset while tuning a single knob for best performance. The TH555A input capacitance is shunted with a $25 \mathrm{Ohm}$ resistor, and the pair of these is driven out of phase by a Ruthroff $1: 1$ balun on a 4C4 ferrite core. Tuning is broadly accomplished with a shunt inductor at the input, reflected through the balun, placing resonance at about $4 \mathrm{MHz}$, and presenting a reasonable match at both 2.8 and $5.6 \mathrm{MHz}$ for drive from a solid state amplifier. This allowed for testing at dual frequencies later.

The output power in this stage is $100 \mathrm{~kW}$ peak per tube, with $5 \%$ duty factor. The quiescent plate current during the bias pulse is 40 Amperes DC per tetrode, in class A. Drive requirements are provided by a single ENI A300 linear amplifier, rated at 300 Watts. IPA stage power gain is approximately $27 \mathrm{~dB}$. 


\subsection{RF Interconnection}

The front of the FPA cabinet was modified to accept a pair of shielded "feeders" connected to the grid resistors. They physically resemble $108 \mathrm{Ohm}$ air insulated coaxial line. The outer conductor is a $7.69 \mathrm{~cm}$ diameter flexible section made from a Myatt corrugated line, with the inner being a $1.27 \mathrm{~cm}$ diameter solid brass rod. At the FPA end, the rod has a gold-plated tip which mates with a high reliability receptacle, made by Hypertronics Corp. A funnel-shaped insulator guides the pin into the hole for
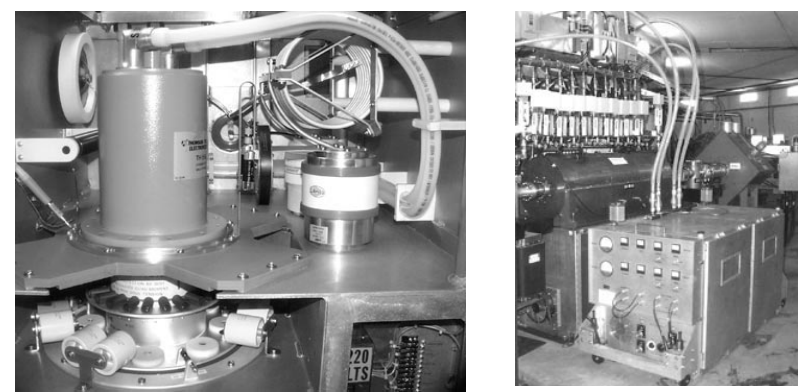

Figure $2 \mathrm{a}$ and $2 \mathrm{~b}$. Interior of IPA, and System Installation

blind alignment. The line was optimized to minimize inception of $60 \mathrm{~Hz}$ AC corona, having a maximum gradient of $8.8 \mathrm{kV} / \mathrm{cm}$ at the buncher peak voltage. Figure $2 \mathrm{~b}$ shows the installed IPA along with the FPA and cavity.

\subsection{Final Power Amplifier Operating Point}

The maximum current in each FPA triode for class A operation is chosen to handle half the peak beam current plus losses. The quiescent current is 40 Amperes, with the same plate voltage as the IPA, $15 \mathrm{kV}$ DC. Bias pulsing is used to keep the DC current under 3 Amperes. Cavity losses are less than $2 \mathrm{~kW}$ at $5 \%$ duty factor.

\subsection{New Gap, Bias and Coupling Loop}

The original gap had a pneumatic-actuated shorting sleeve on one side. It was no longer used, and was a source of unwanted stray capacitance to ground on that side only. Further inspection found the shorting sleeve inside the beam pipe in poor condition, with burned wiping contacts due to arcing. This mechanism was removed and replaced with a symmetric gap structure for electrical balance.

The original biasing system for the ferrite was a pair of parallel copper loops for DC, with RF bypassing capacitors to ground. DC was shunt fed through a large RF choke inductor external to the cavity. There was conducted RF leakage into the power cables. With the upgrade, the bias loops were converted to a "figure-eight" arrangement with RF coupling and DC on the same busswork. Figure 3 shows the arrangement. Since the loops are now in series, the DC current is half the old value for resonance at $2.8 \mathrm{MHz}$, allowing the same power supply to push resonance as high as $5 \mathrm{MHz}$ for testing. There is very little residual RF at the bias feed point, with this balanced hookup. A small common-mode ferrite choke is used as an RFI filter, encompassing both DC conductors to prevent saturation. The RF coupling capacitors at the ends are de-Q'd with a low inductance resistor of $0.1 \mathrm{Ohms}$, composed of 100 individual carbon resistors in parallel on copper plates. These loops tightly couple the halves of the cavity in push-pull. Modeling and testing demonstrated improved output compliance. The bussbars are free of stray resonances to $30 \mathrm{MHz}$.

\subsection{Low Level RF Feedback Control}

The amplitude of the RF buncher is ramped with injection. A feedback control system is driven from a differential gap voltage sample. In the upgraded system, the loop bandwidth was significantly increased and an integral gain term was incorporated. An active detector was designed to control at a lower gap voltage, about 500 volts versus $2 \mathrm{kV}$ for the original controller. The original system had no phase control. With the addition of the tuned IPA, a phase feedback loop was designed. Using a $\log$ amp as a limiter, a double balanced mixer is used to derive a phase signal, and a steerable phase modulator is controlled. This reduced open loop phase slew during the amplitude ramp from 18 to 9 degrees, as well as locking the phase to a reference. Beam effects on the buncher will be discussed in the companion paper [2].

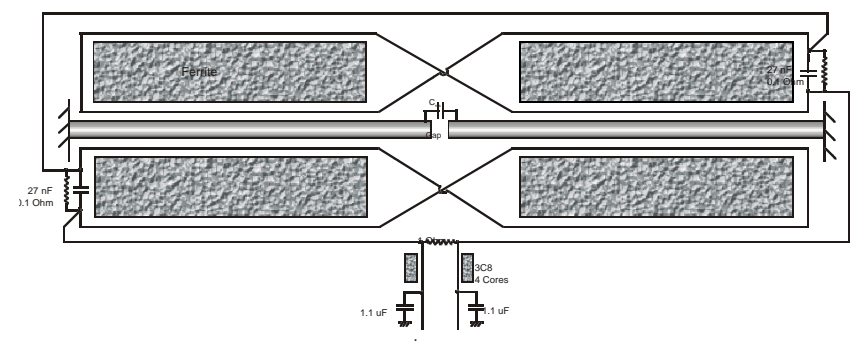

Figure 3. Figure-Eight Coupling and DC Bias Bussbars

\section{REFERENCES}

[1] R. Macek, et al. "Overview of Progress on the Improvement Projects for the LANSCE Accelerator and Target Facilities", Proceedings of 1997 Particle Accelerator Conference, Vancouver, BC.

[2] J. Lyles, A. Browman, R. Kustom, "Commissioning of The Upgraded RF Buncher at the LANSCE Proton Storage Ring", Proceedings of 1999 Particle Accelerator Conference, New York, NY.

[3] J. Lyles, A. Browman, J. Doss, "RF Buncher Upgrade for SPSS Enhancement Project", PSR Tech Note 97-004.

[4] T. Hardek, “A Low Impedance, 2.8 MHz, Pulsed Bunching System for the Los Alamos Proton Storage Ring", Sixteenth Power Modulator Symposium, Arlington, VA. June 18-20, 1984.

[5] S. Giordano, M. Puglisi, "A Cathode Follower Power Amplifier", IEEE Transactions on Nuclear Science, Vol. NS-30, No. 4, August, 1983.

[6] F. Clapp, "Some Aspects of Cathode-Follower Design at Radio Frequencies", Proceedings of the I.R.E., Waves and Electrons Section, August 1949. 